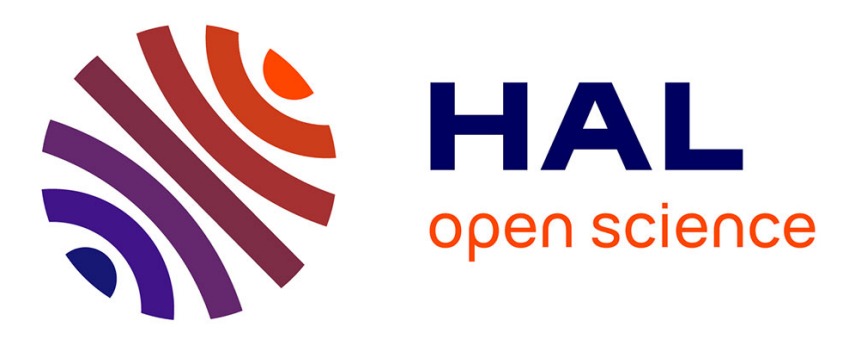

\title{
Signatures of Wigner localization in one-dimensional systems
}

\author{
Alejandro Diaz-Marquez, Stefano Battaglia, Gian Luigi Bendazzoli, Stefano \\ Evangelisti, Thierry Leininger, Arjan Berger
}

\section{To cite this version:}

Alejandro Diaz-Marquez, Stefano Battaglia, Gian Luigi Bendazzoli, Stefano Evangelisti, Thierry Leininger, et al.. Signatures of Wigner localization in one-dimensional systems. Journal of Chemical Physics, 2018, 148 (12), pp.124103. 10.1063/1.5017118 . hal-01788389

\section{HAL Id: hal-01788389 \\ https://hal.science/hal-01788389}

Submitted on 18 Sep 2018

HAL is a multi-disciplinary open access archive for the deposit and dissemination of scientific research documents, whether they are published or not. The documents may come from teaching and research institutions in France or abroad, or from public or private research centers.
L'archive ouverte pluridisciplinaire HAL, est destinée au dépôt et à la diffusion de documents scientifiques de niveau recherche, publiés ou non, émanant des établissements d'enseignement et de recherche français ou étrangers, des laboratoires publics ou privés. 


\section{ACCEPTED MANUSCRIPT}

This article may be downloaded for personal use only. Any other use requires prior permission of the author and AIP Publishing. The following article appeared in A. Diaz-Marquez et al., J. Chem. Phys. 148, 124103 (2018) and may be found at https://doi.org/10.1063/1.5017118

DOI: https://doi.org/10.1063/1.5017118 


\section{Signatures of Wigner localization in one-dimensional systems}

Alejandro Diaz-Marquez, ${ }^{1}$ Stefano Battaglia, ${ }^{1,2}$ Gian Luigi Bendazzoli, ${ }^{3}$ Stefano

Evangelisti, ${ }^{1,}$ a) Thierry Leininger, ${ }^{1}$ and J. A. Berger ${ }^{1,4, b)}$

1) Laboratoire de Chimie et Physique Quantiques, IRSAMC, CNRS, Université

Toulouse III - Paul Sabatier, 31062 Toulouse, France

2) Dipartimento di Chimica, Biologia e Biotecnologie, Università degli Studi di

Perugia, Via Elce di Sotto 8, 06123 Perugia, Italy

3) Università degli studi di Bologna, Viale Risorgimento 4, I-40136 Bologna, Italy

4) European Theoretical Spectroscopy Facility (ETSF)

(Dated: September 11, 2018)

We propose a simple and efficient approach to study Wigner localization in onedimensional systems using ab initio theory. In particular, we propose a suitable basis for the study of localization which consists of equally spaced overlapping gaussians. We illustrate our approach with full-configuration interaction which yields exact results for a given basis set. With our approach we were able to study up to 8 electrons with full-configuration interaction. Finally, we propose the total-position spread tensor and the total electron entropy as convenient quantities to obtain signatures of Wigner localization.

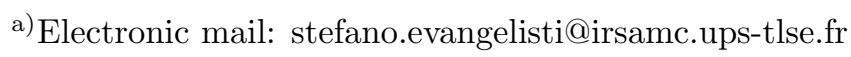

b) Electronic mail: arjan.berger@irsamc.ups-tlse.fr 


\section{INTRODUCTION}

When electrons are confined to one dimension (1D) they exhibit remarkable physics. Well-known examples are the quantization of the conductance ${ }^{1,2}$, fractional conductance ${ }^{3,4}$, spin-charge separation $^{5-8}$, and the enhanced localization of electrons at low densities ${ }^{8,9}$. Due to the latter property these 1D systems have been shown to be interesting candidates for applications in quantum computation ${ }^{10,11}$. When only few electrons are involved, these 1D systems are often referred to as Wigner molecules owing to the similarity with Wigner crystallization $^{12,13}$. Recently, a 2-electron Wigner molecule has been observed experimentally ${ }^{14}$.

To treat Wigner molecules numerically two problems have to be overcome: i) The nonintegrable singularity of the $1 \mathrm{D}$ Coulomb potential in the origin ${ }^{15-18}$. ii) The lack of a suitable basis in which to express the many-body wave function. In particular, the two most common types of basis sets, i.e, atom-centered basis functions and plane waves, are not convenient. The first problem can be circumvented by modeling the system in three dimensions, in which there is no problem to integrate the Coulomb potential, as a cylinder of infinite length and a radius so small that the transverse motion of the electrons is frozen in the lowest energy state ${ }^{19}$. In this work we will address the second problem.

Theoretically, Wigner localization can be studied either through phenomenological effective Hamiltonians ${ }^{14}$, or by using the non-relativistic electronic ab initio Hamiltonian. In almost all studies on finite Wigner systems the former option is chosen, (see, e.g., Ref. ${ }^{20,21}$ ) and the phenomenological effective Hamiltonian contains an external potential, often having a harmonic component, to confine the system. The molecular orbitals (MO) of the system are then obtained by diagonalizing a one-electron Hamiltonian, given by the kinetic energy plus the confining potential. This is an appropriate strategy for few electrons in a harmonic well. However, it can lead to computational difficulties if the number of electrons (and hence the number of MO's) is large, or if the electrons are confined in a spatial region by a very steep potential. This is the case of electrons in boxes of different shapes. In fact, the MO's quickly become strongly oscillating (in 1D systems, the $n$-th excited state has $n$ nodes), which can be a source of numerical instabilities.

Therefore, in this work we propose to study the non-relativistic electronic ab initio Hamiltonian without any explicit external potential. Instead, we use a set of equidistant localized orbitals, that are distributed in the region of the space where the electrons are confined. 
This induces an implicit confining potential, which originates from the basis set itself. We choose the localized orbitals as simple $1 s$ gaussian orbitals with a unique exponent and electric neutrality is guaranteed by a background of equidistant fractional positive charges. The main advantage of our approach is that, with minor modifications, all standard quantumchemistry codes can be used to study Wigner localization, since they all deal with gaussian $\mathrm{MO}$ and the positive background can be realized by placing fractional point charges in the region occupied by the orbitals. Any quantum chemistry approach can thus be used to study Wigner localization. However, since Wigner molecules of few electrons can be treated exactly, we will illustrate our approach mainly with full-configuration interaction (FCI).

Finally, in this work we propose several indicators for the study of localization. Apart from the electron density, we find signatures of Wigner localization also in the total-position spread tensor, which is linked to the conductivity, as well as the total electron entropy.

The manuscript is organized as follows. In section II we describe the formalism of our approach, while in section III we discuss the computational details. In section IV we show and discuss our results and we draw our conclusions in section V.

\section{FORMALISM}

In this work we make use of quantum-chemistry approaches to study the localization of electrons confined in one dimension. In particular, we use the restricted open-shell HartreeFock (ROHF) method and Full Configuration Interaction (FCI) in order to expand the wave function. The FCI expansion is obtained by considering all the Slater determinants that can be generated within a given set of one-electron functions (spinorbitals). The Hamiltonian is the ab initio non-relativistic electronic operator describing the kinetic energy and the Coulomb interactions which consists of the electron-background attraction, and the electronelectron repulsion.

We choose the orbitals to be equally spaced $s$-type gaussian orbitals with a unique exponent, and whose equally spaced centers are placed in the region of space where the electrons are confined (in our particular case we choose the $z$ axis). We note that such a 1D arrangement of gaussian orbitals realizes the regularization procedure that is required to treat a 1D Coulomb potential ${ }^{19}$. In fact, the transverse (i.e. orthogonal to the $z$ axis) part of the wavefunction has a gaussian form, as it happens for an electron confined in a harmonic radial 
potential. It should be noticed that the present approach is able to recover the (analytical) high-density results of the Fermi-gas region, showing the viability of this quantum-chemistry treatment of a set of confined electrons.

The most straightforward way to investigate electron localization in a system with open boundary conditions (OBC), i.e., a system without translational invariance, is by computing its electron density, given by the diagonal part of the one-body reduced density matrix. Besides the density, we study two other quantities that give information about electron localization and correlation: the total-position spread (TPS) tensor $\boldsymbol{\Lambda}$, and the total electronic entropy $S$. The TPS is defined as the second moment cumulant of the total position operator $\hat{\mathbf{R}}$ according to

$$
\boldsymbol{\Lambda}=\left\langle\Psi\left|\hat{\mathbf{R}}^{2}\right| \Psi\right\rangle-\langle\Psi|\hat{\mathbf{R}}| \Psi\rangle^{2}
$$

where $\hat{\mathbf{R}}$ is defined as

$$
\hat{\mathbf{R}}=\sum_{\mu=1}^{N} \mathbf{r}_{\mu}
$$

with $N$ the number of electrons. We note that the purpose of the second term in Eq. (1) is to ensure gauge invariance. The per-electron TPS tensor is the localization tensor $\lambda=\Lambda / N$ which was introduced by Resta and co-workers ${ }^{22,23}$ following an original idea of Kohn ${ }^{24}$ (see also Ref..$^{25}$ ). The localization tensor differentiates the behavior of conductors and insulators: in the limit $N \rightarrow \infty$ it diverges for conductors while it remains finite in the case of insulators.

For this reason, a qualitatively different behavior of the localization tensor is expected in the Fermi-liquid and Wigner-crystal regimes of the electron gas. The localization tensor has been used to investigate the metallic behavior of finite clusters, both at $a b$ initio ${ }^{26-28}$ and semi-empirical ${ }^{29-34}$ level.

Electron correlation can be related to the natural occupation numbers ${ }^{35}$. Therefore, the electron entropy has been proposed as a measure of the presence of correlation in a system $^{36,37}$. In the case of a pure state described by a wavefunction $\Psi$, the particle-hole entropy is defined as:

$$
S=-\sum_{j=1}^{M}\left[n_{j} \ln n_{j}+\left(1-n_{j}\right) \ln \left(1-n_{j}\right)\right]
$$

where the sum runs over the $M$ natural spinorbitals of $\Psi$, and $n_{j}$ is the occupation number 
of spinorbital $j$. The first and second terms in the summation are the electron and hole contributions, respectively, to the total entropy. While the entropy of a single determinant is zero, the entropy has its maximum value when all the spinorbitals have equal occupation numbers. For this reason, we expect a very small entropy for the Fermi gas and a much larger entropy in the Wigner regime. In particular, this will be the case for the $S_{z}=0$ wave functions, because of the large number of Slater determinants that contribute to the wave function with similar weight.

In analogy with standard quantum-chemistry notation, we will use the expressions "atomic orbital" and "molecular orbital" in order to indicate, respectively, the primitive non-orthogonal orbitals that are used as a basis set in which we expand the wave function, and the orbitals that result from the diagonalization of the Hamiltonian of the system. As is common practice in quantum chemistry, we will use gaussian orbitals for the primitive basis set. In this work, the primitive atomic orbitals (AO), which we use to expand the molecular orbitals (MO), are normalized three-dimensional $s$-type gaussian functions, all having a single common exponent $\alpha$ :

$$
\phi_{\alpha, \mathbf{r}_{0}}(\mathbf{r})=(2 \alpha / \pi)^{\frac{3}{4}} \exp \left(-\alpha\left\|\mathbf{r}-\mathbf{r}_{0}\right\|^{2}\right)
$$

where $\mathbf{r}_{0}$ is the position of the center of the gaussian orbital.

In order to describe a smoothly varying function in an accurate way, two gaussians must be relatively close together. Therefore, the overlap between two normalized gaussians plays a crucial role in defining a suitable expansion basis set. The overlap $\mathcal{S}$ between two normalized gaussians having a common exponent $\alpha$ and with a distance $\delta$ between their two centers depends on the dimensionless quantity $\xi=\alpha \delta^{2}$ only. It is given by the expression

$$
\mathcal{S}(\alpha, d)=\mathcal{S}(\xi)=\exp (-\xi / 2)
$$

A set of sufficiently overlapping gaussian orbitals is able to describe a smoothly varying function in a very accurate way. In order to obtain an accurate description of the wavefunction, two neighbouring gaussians must have a large overlap and, therefore, $\xi$ must be small. In particular, if $\xi>2$ the sum of two gaussians which have the same weight yields a function with two peaks, which is unsuitable to provide a smooth description of a wave function. On 
the other hand, if $\xi$ is too small, and hence the overlap too large, one runs into the problem of a quasi-linear dependent basis set, which can lead to numerical instabilities. In this work we choose to work with a fixed value for $\xi$. This means that the width of the gaussian is proportional to the distance between two neighboring centers. More precisely, we we will use the value $\xi=1$. Previous investigation have shown that this value can recover the energy levels of, for example, a harmonic oscillator with a very high accuracy ${ }^{38}$.

\section{COMPUTATIONAL DETAILS}

In this work, we used $g+1$ equally spaced gaussians in order to describe a single electron confined in a $1 \mathrm{D}$ region of the space. If the electron is placed in a region of the $z$ axis of length $L$, namely $-L / 2<z<L / 2$, the first gaussian will be placed at $z=-L / 2$ and the last one at $z=L / 2$. In such a way, the distance between two centers is given by $\delta=L / g$. In Fig. 1, the gaussians used to describe a single electron are shown for the case $g=12$ and $\xi=1$. We note that, since we choose $\xi$ to be a constant, the length units on the bottom axis in Fig. 1 are arbitrary. By analogy, a total of $N g+1$ orbitals is needed to describe $N$ electrons in a region of length $N L$. In numerical calculations the choice of $g$ plays a crucial role, since the size of the FCI space becomes quickly unmanageable for large values of $\mathrm{Ng}$. In this work, we performed benchmark FCI calculations for two and four electrons with $g=12$. These calculations were used to assess the quality of results obtained with $g=4$. We verified that by going from $g=12$ to $g=4$, the numerical differences are negligeable and the qualitative overall picture is left unchanged. In this way, by using such a small value of $g$, we were able to study systems with 2 to 8 electrons within the FCI method.

We used a set of pointlike fractional positive charges, uniformly distributed on the $z$ axis, in order to simulate the jellium uniform background. The first and last charges of the set coincide with the centers of the first and last gaussian. The pointlike charges have been chosen with the same procedure as the gaussian centers. In particular, we used a number of $q+1$ charges for a segment of length $L$, and hence $N q+1$ for the $N$-electron system. The value of these charges are chosen in such a way to have a system that is globally neutral. This means that each charge has a value of $+N /(N q+1)$ in atomic units. We verified that a value of $q$ of the order of ten is needed in order to have a converged electronic wavefunction. For this reason, and to have a maximum number of the point charges coincide with the 
gaussian centers for the case $g=12$, all the calculations presented in this work have been obtained by setting $q=12$.

All the calculations reported in this work have been performed with the packages Neptunus $^{39-41}$ and Molpro ${ }^{42,43}$. The former is an FCI algorithm that uses the MO integrals computed by the DALTON software package ${ }^{44}$. It has been used for calculations with $m=2,3,4$ and $g=12$. For larger number of electrons, the FCI wavefunction was obtained by using the CAS-SCF algorithm of the Molpro package ${ }^{45,46}$.

\section{RESULTS AND DISCUSSION}

We express all our results as a function of the formal mean distance $d$ between two electrons, $d=L / N$. We note that $d$ should not to be confused with the mean value of the interelectronic-distance operator. The mean distance $d$ is the inverse of the electron density and twice the Wigner-Seitz radius $r_{s}$ of the $1 \mathrm{D}$ system. We investigated the behavior of the system from an extremely high-density regime $(d=0.01$ Bohr $)$ to a very low-density regime $(d=100$ Bohr $)$.

In this work, we focus on the following two spin wave functions,

1. the high-spin (HS) solution, $S_{z}=N / 2$, having a maximum spin multiplicity equal to $N+1$.

2. the low-spin (LS) solution $\left(S_{z}=0\right.$ for an even number of electrons, having spin multiplicity equal to one

To verify our computational approach, we first consider the high-density limit. Since the electron-electron repulsion, and more generally all the Coulomb interactions present in the system, scale as $1 / d$, while the kinetic energy of an individual electron scales as $1 / d^{2}$, in the high-density limit, $d \rightarrow 0$, the Coulomb interaction becomes negligeable with respect to the kinetic contribution. Therefore the electrons behave as a gas of free non-interacting particles and the wavefunction of the system becomes the wavefunction of a set of free fermions in a 1D box, whose behavior is analytically known. For this reason, it is possible to check the quality of our computational approach by comparing our numerical results with analytical expressions. 
In Fig. 2 the energy difference per electron between the HS and LS solution as a function of the mean distance $d$ is reported on a bilogarithmic scale. The energy of the LS wave function is below the HS wave function for all values of $d$ in the considered interval. At high density, where the system is well described by a Fermi gas, an analytical expression for the gap can be derived from the energy levels of a particle in a 1D box of length $L$. They are given by the expression $\epsilon_{n}=n^{2} \pi^{2} / 2 L^{2}$. By summing the levels of the occupied orbitals in the HS and LS cases, and using the identity $\sum_{n=1}^{N} n^{2}=N(N+1)(2 N+1) / 6 \simeq N^{3} / 3$ for large $N$, we obtain, for large values of $n$, the following asymptotic expression

$$
\Delta_{E} / N=\left(E_{H S}-E_{L S}\right) / N=\pi^{2} / 8 d^{2} .
$$

We see that in the high-density limit, $d \rightarrow 0$, our numerical results agree with the analytical result. We note that the differences between the various curves for different values of $N$ are not appreciable due to the logarithmic scale.

Now that we have validated our approach we will study the electron localization in the low-density regime. In Figs. 3-6 we report the FCI electron density of the system with six electrons, for both the LS and HS wavefunctions, and for four different values of $d: 0.1,1.0$, 10.0, and 100.0 Bohr. We see that for $d=0.1$ and $d=1.0$, the density is almost a constant, and similar to the density of six non-interacting electrons in a one-dimensional box. Indeed, six peaks are shown in the HS case, and only three (because of the double occupation of the orbitals) in the LS case. Between $d=1.0$ and $d=10.0$ Bohr, the charge distribution completely changes: In the latter case there are six peaks in both the HS and LS cases, separated by deep valleys. At the largest distance shown, $d=100.0$ Bohr, the density at the bottom of the valleys is almost vanishing, and there is no noticeable difference between the HS and LS densities.

Instead of studying the density for various values of $d$ it would be more practical to study a single function of $d$ that contains similar information about the electron localization. In Fig. 7 we report the values of the HS localization tensor $\lambda$ for both ROHF and FCI. In order to compare results related to different box sizes, the localization tensor has been divided by $d^{2}$. We note that $\lambda / d^{2}$ is a dimensionless quantity. There are two important conclusions that can be drawn. First, Fig. 7 shows a striking decrease of $\lambda / d^{2}$ in the region between $d=1.0$ and $d=10.0$ Bohr, which corresponds to the localization that we observed for the 
electronic density. Therefore, the sudden decrease of $\lambda / d^{2}$ can be considered a signature of Wigner localization. Second, Fig. 7 demonstrates the importance of the accurate treatment of electron correlation in the low-density regime. At high densities, the ROHF and FCI results coincide and become equal to the free-electron high-density limit, as they should. Moreover, in the high-density regime, $d<1.0$ Bohr, the value of $\lambda / d^{2}$ for both ROHF and FCI is roughly proportional to $N$, as one would expect for a conducting electron gas. Instead, in the low-density regime the ROHF and FCI results are completely different. The ROHF localization tensor becomes independent of the number of electrons for large $d$, which would indicate an insulating behavior. However, the FCI localization tensor, i.e., $\lambda / d^{2}$, is proportional to $N$ also in the low-density regime, indicating the presence of conduction. Therefore, despite the fact that the electrons are localized, the localization tensor shows a behavior that corresponds to a conducting state at least up to $d=50$.

In Fig. 8 the LS and HS FCI values of the localization tensor are compared. For high densities, the LS values are considerably larger than the HS ones, since for the latter case the electron mobility is reduced due to Pauli repulsion. Beyond $d=10$ Bohr, however, the two sets of curves become perfectly superposed, as one would expect for localized electrons that differ only in their spin coupling.

In Fig. 9 we report the electronic total, i.e., particle+hole, entropy per electron as a function of the number of electrons for the lowest HS and LS states. We note that, since the entropy, in general, depends on the spin projection, for the HS state we report the values for the $S_{z}=0$ and $S_{z}=\max$ components. In all cases the entropy increases between $d=1.0$ and $d=10.0$ Bohr, corresponding to the region in which the electrons become localized. Therefore, the sudden increase of the total entropy can also be considered as a signature of Wigner localization. As mentioned before, the entropy of a single Slater determinant is identically zero, while a nonzero value for $S / N$ means that some kind of correlation is present.

Electron correlation is traditionally split into a dynamical and a non-dynamical (or static) part. Although there is not a clear-cut distinction between the two components, several definitions have been proposed ${ }^{47-50}$. We do not want to give an additional definition here. However, broadly speaking, one can say that non-dynamical correlation is a phenomenon associated to the presence of many roughly equivalent Slater determinants in the zero-order description of the wave function. The dynamical part, on the other hand, is related to the 
huge number of determinants (each one giving a small contribution) that are needed for a correct description of the Coulomb hole in the wave function. In the following discussion we will use this qualitative distinction between dynamical and non-dynamical correlation.

It is interesting to relate the entropy, as shown in Fig. 9, to the presence of the two types of correlation. Both the $\operatorname{LS}$ and $\operatorname{HS}\left(S_{z}=\max \right)$ FCI entropies vanish for small values of $d$, i.e., in the Fermi-gas regime, since in both cases the wave function is well represented by the ROHF Slater determinant. This situation corresponds to the absence of both types of correlations. The $\operatorname{HS}\left(S_{z}=\max \right)$ entropy, on the other hand, goes to a relatively large finite limit when $d \rightarrow 0$. This is due to the fact that many Slater determinants are needed to describe the wave function despite the fact that we are in the large-density limit where the Coulomb interactions become negligible with respect to the kinetic energy. This corresponds to the presence of non-dynamical correlation, since dynamical correlation is absent. It can be verified from Eq. (3) that the total entropy of $N$ electrons evenly distributed into $2 N$ spinorbitals is given by $2 N \ln 2$. Therefore, the entropy of the $\operatorname{HS}\left(S_{z}=0\right)$ state converges towards $2 N \ln 2$ in the limit $d \rightarrow 0$ and the entropy per electron converges to $S / N=2 \ln 2=$ 1.386294 in this limit. From Fig. 9 we see that the entropies for the LS and the $\operatorname{HS}\left(S_{z}=0\right)$ state become identical for medium-large distances, when each electron tends to be well localized in space. In this case, the $S_{z}=0$ wave functions are dominated by determinants with localized electrons having an equal probability of $\alpha$ and $\beta$ spins, and the value of $2 \ln 2$ can be interpreted as the entropy per electron due to non-dynamical correlation. The difference $S / N-2 \ln 2$ can therefore be interpreted as the contribution to the entropy per electron that is due to dynamical correlation.

Because of the $D_{\infty h}$ symmetry of the systems we study here, the natural orbitals are partly delocalized symmetry-adapted pairs, having $g$ and $u$ symmetries. However, by performing a simple combination of the orbitals that correspond to $g$ and $u$ symmetries, we obtain completely localized orbitals. The localized orbitals that account for the overwhelming part of the occupation numbers are symmetric (the most important ones) and antisymmetric orbitals. In the language of three-dimensional atomic orbitals, they correspond to $s$ - and p-type orbitals, respectively. In Fig. 10, localized orbitals corresponding to the four largest occupation numbers are shown. They are s-type orbitals, and account for more than $90 \%$ of the total orbital occupation numbers. In Fig. 11, localized orbitals corresponding to the four next-largest occupation numbers are shown. They are $p$-type orbitals which are located 
in the same region as the $s$-type orbitals.

\section{CONCLUSIONS}

We studied the behavior of a small number of electrons confined to a quasi-1D arrangement by the basis set used to expand their wave function. At high density the wave function of the system is similar to the one of a free-electron system. However, by lowering the density there is a complete change of the wave function structure. The electrons change from a state where they are essentially delocalized, and well described by a single Slater determinant, to a state where they are localized at fixed space positions. This is true for both the high-spin and low-spin states.

We obtained a deeper insight on the nature of electron localization by studying the localization tensor and the electron entropy. Indeed, by lowering the electron density we observe a sudden growth of the entropy, and a corresponding drop of the position spread in the density regime where the electrons localize. The drop of the position spread indicates a reduced mobility of the electrons with respect to the Fermi-gas solution. This behavior is a signature of Wigner localization and is reminiscent of that of the Wigner crystallization in finite systems (Wigner atoms).

The increase of the electronic entropy also indicates that the low-density state is much more correlated than the Fermi-gas state, which tends to a single determinant (zero correlation) in the limit of very high densities. This behavior is not surprising for the low-spin state, since a large amount of non-dynamical correlation is needed to account for the multideterminant singlet wave function. The picture is less obvious in the case of the high-spin

solution, that is strongly dominated by the high- $\left|S_{z}\right|$ single determinant. Nevertheless, a relatively large amount of dynamical correlation is still present in the localized state, essentially carried by a single $p$-type orbital located on top of each localized $s$ orbital, even at relatively large distances.

The present investigation shows the usefulness of a quantum-chemistry approach to treat a collection of interacting particles confined to a region of space. Finally, we stress that our method is not restricted to the $n$-D jellium model, but could be used to treat electrons in different confining potentials such as quantum dots. 


\section{ACKNOWLEDGEMENTS}

This work has received fundings from the European Union's Horizon 2020 research and innovation programme under the Marie Skłodowska-Curie grant agreement $\mathrm{n}^{\circ} 642294$. The calculations of this work have been partly performed by using the resources of the HPC center CALMIP under the grant 2016-p1048. One of us (ADM) acknowledges the support of the "Theoretical Chemistry and Computational Modelling" (TCCM) Erasmus-Plus Master program. This work was supported by the Programme Investissements d'Avenir under the program ANR-11-IDEX-0002-02, reference ANR-10-LABX-0037-NEXT. The authors would like to thank Paola Gori-Giorgi and Pierre-François Loos for fruitful discussions.

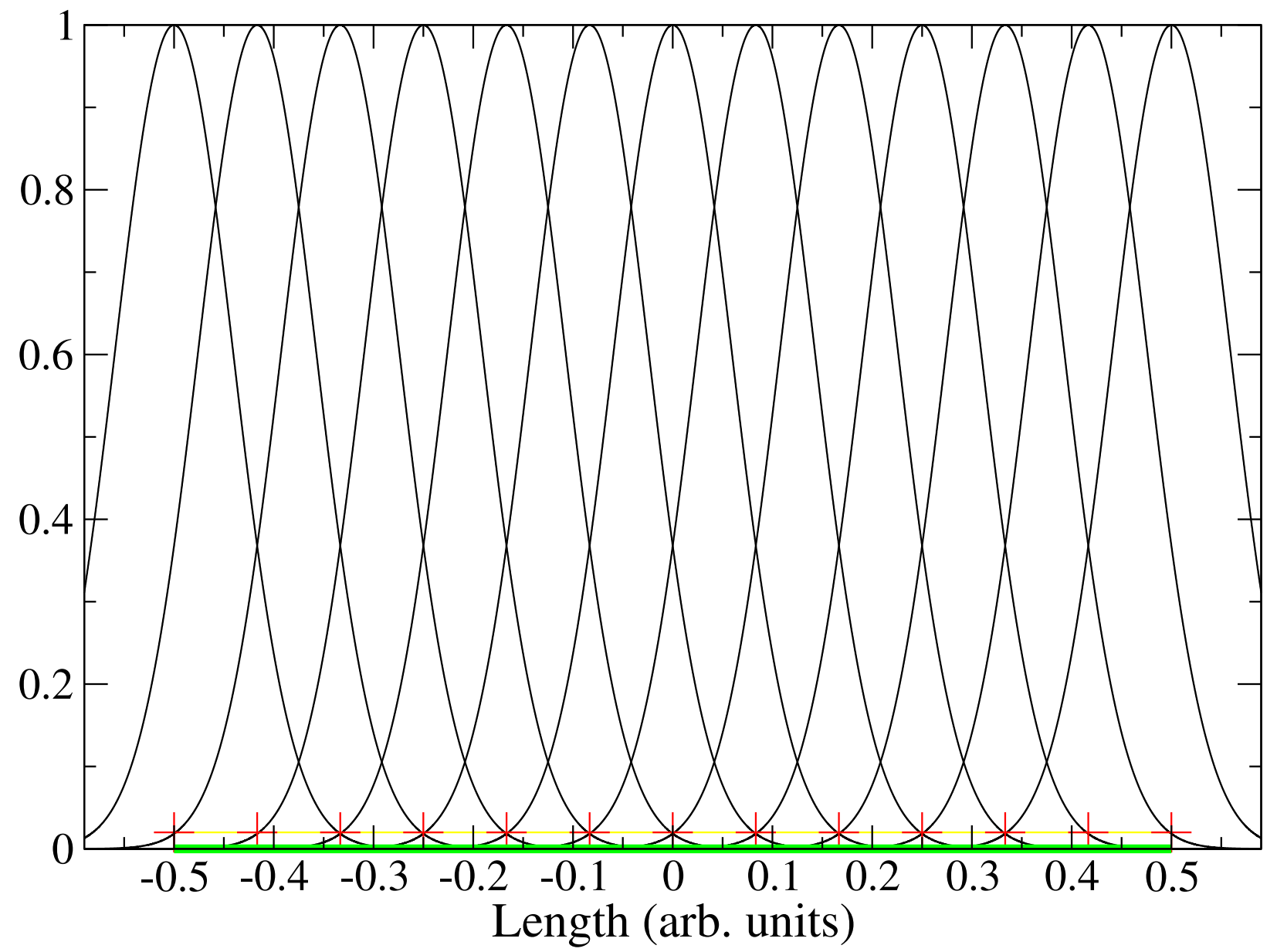

Figure 1. (color online) Example of the tiling of a segment of length $L=1$ with gaussians to describe 1 electron $(g=12)$. Continuous lines (black): the gaussian functions; plus symbols (red): positions of the fractional positive point charges. 


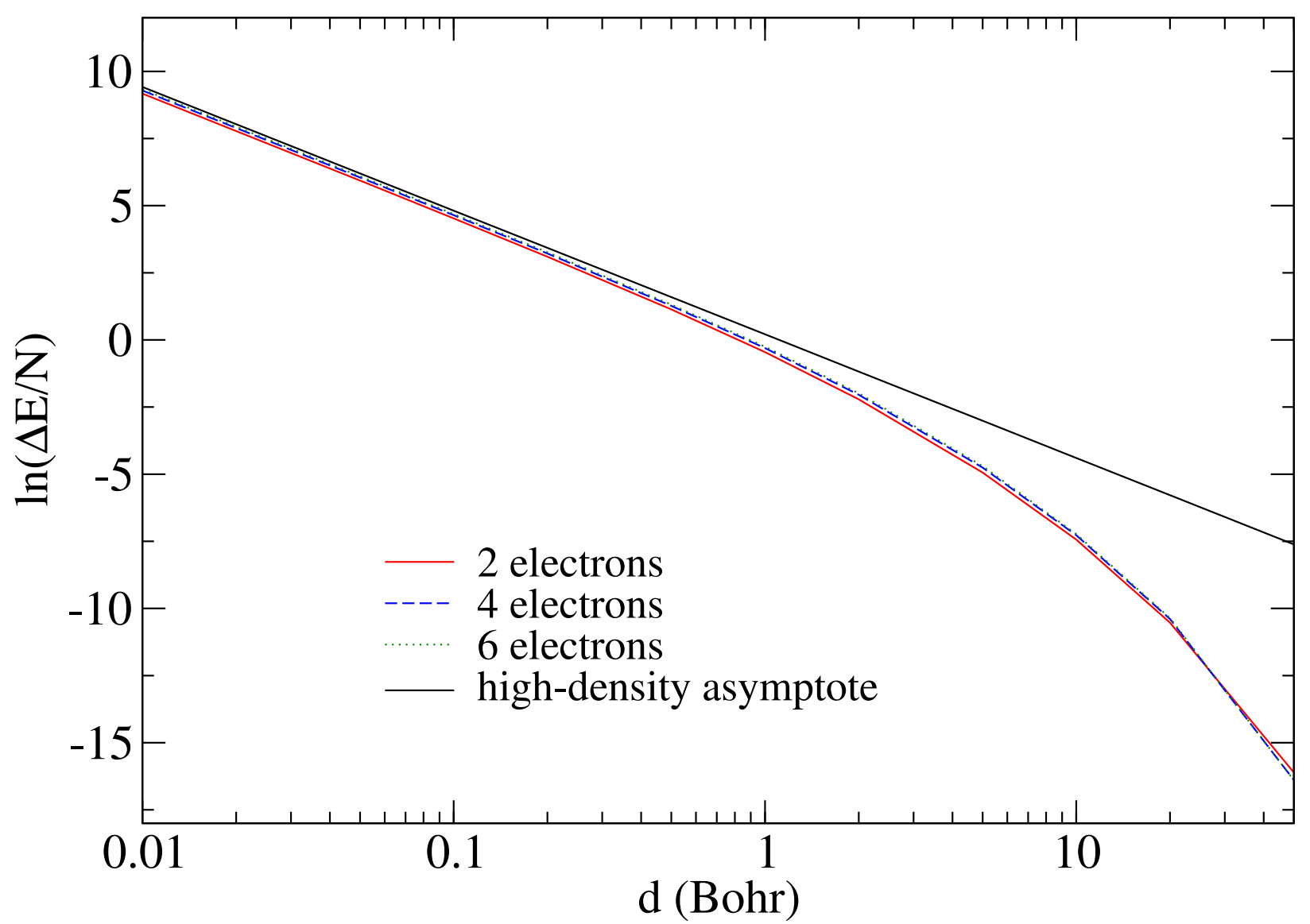

Figure 2. The natural logarithm of the HS-LS energy-difference per electron obtained within FCI as a function of the mean distance $d$ (please note the logarithmic scale). 


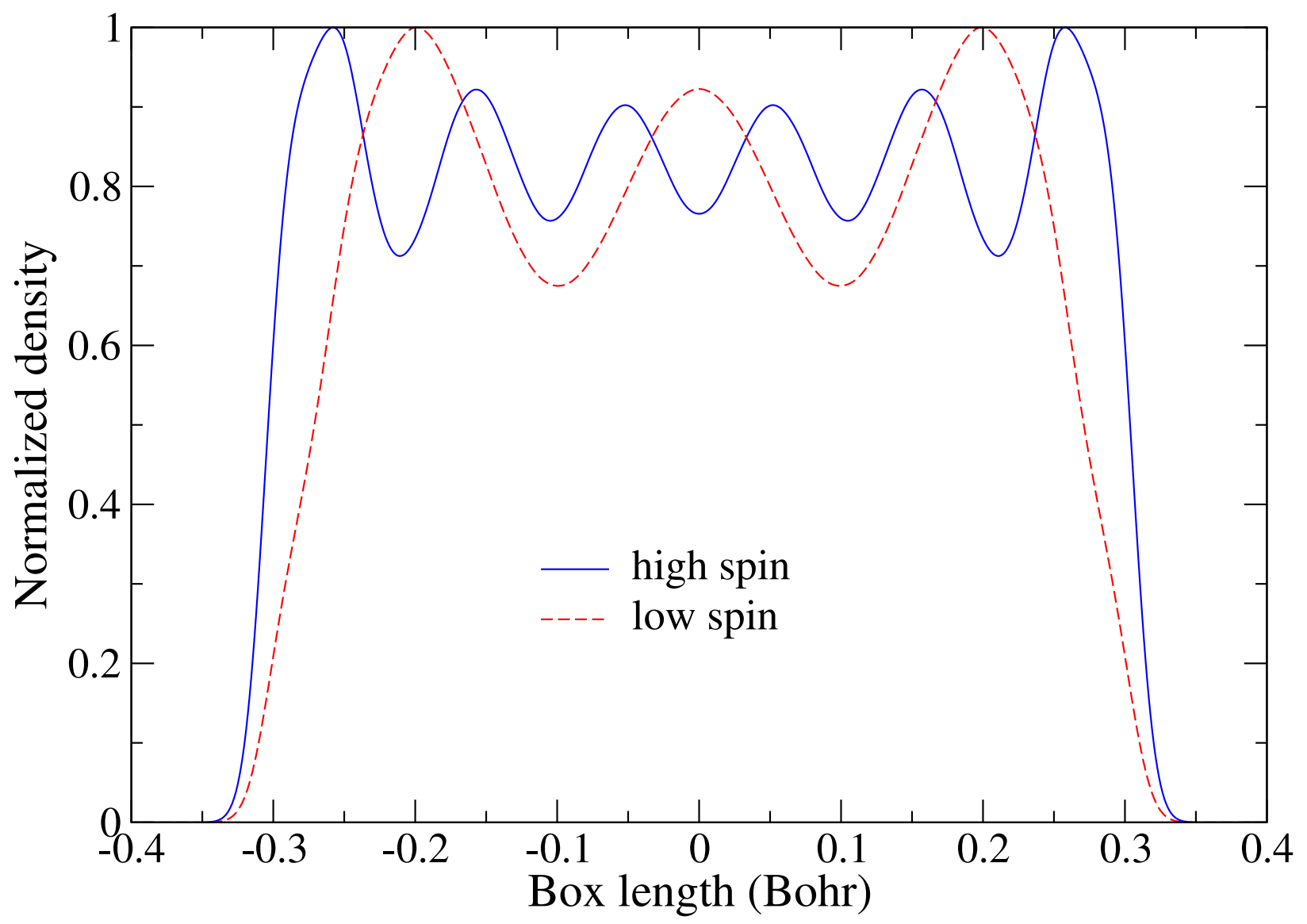

Figure 3. The electron density for $d=0.1$ Bohr. 


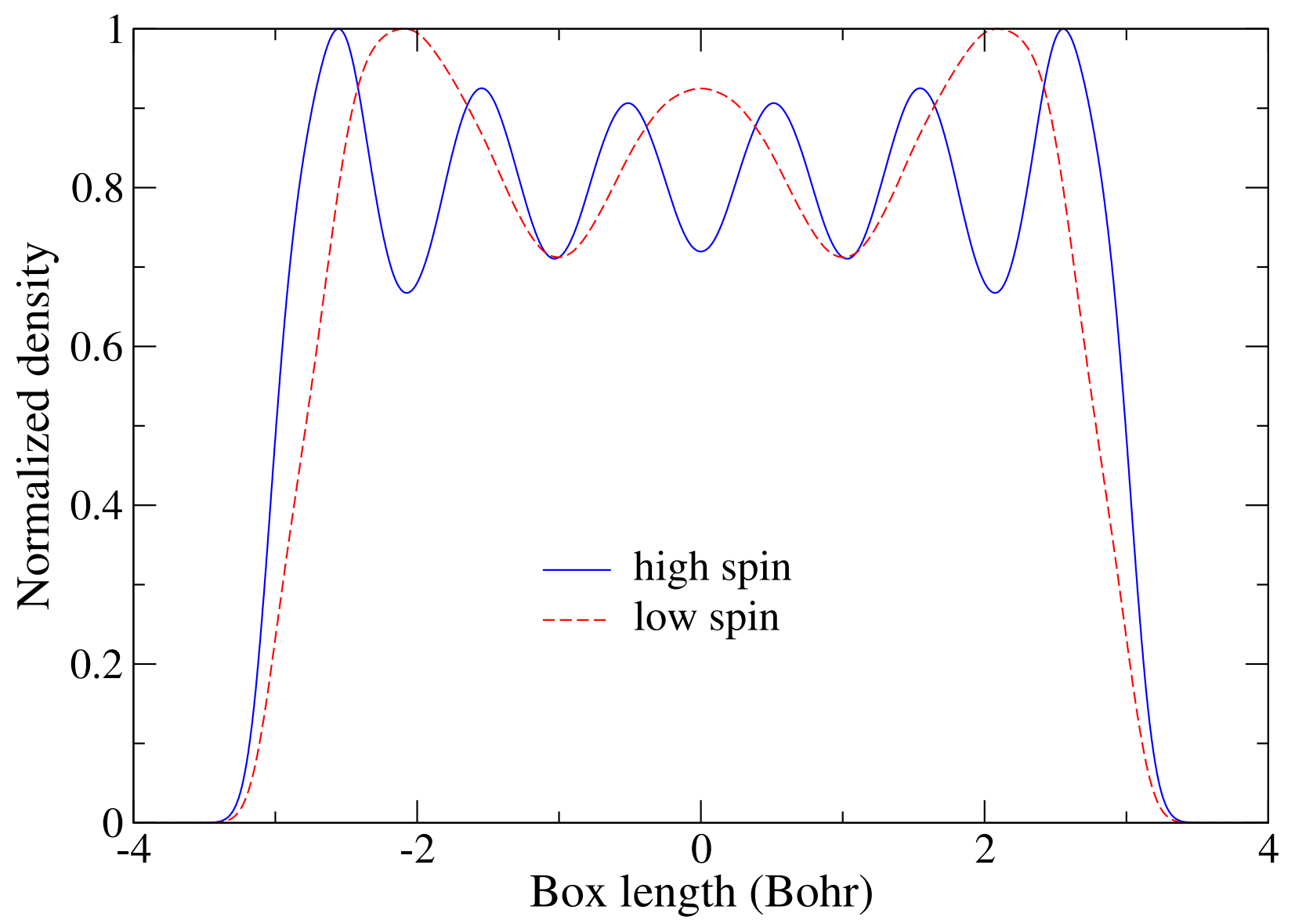

Figure 4. The electron density for $d=1.0$ Bohr. 


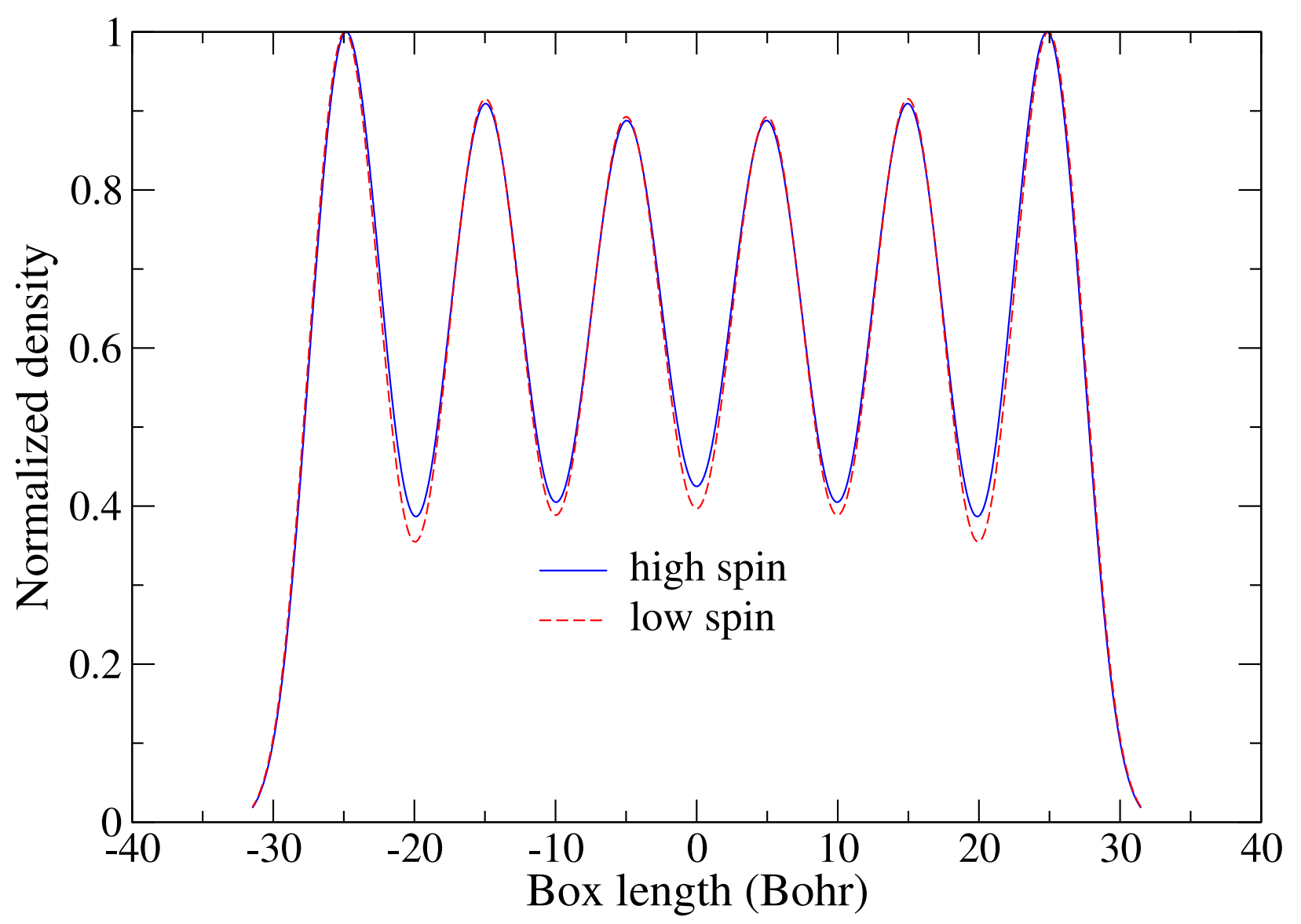

Figure 5. The electron density for $d=10.0$ Bohr. 


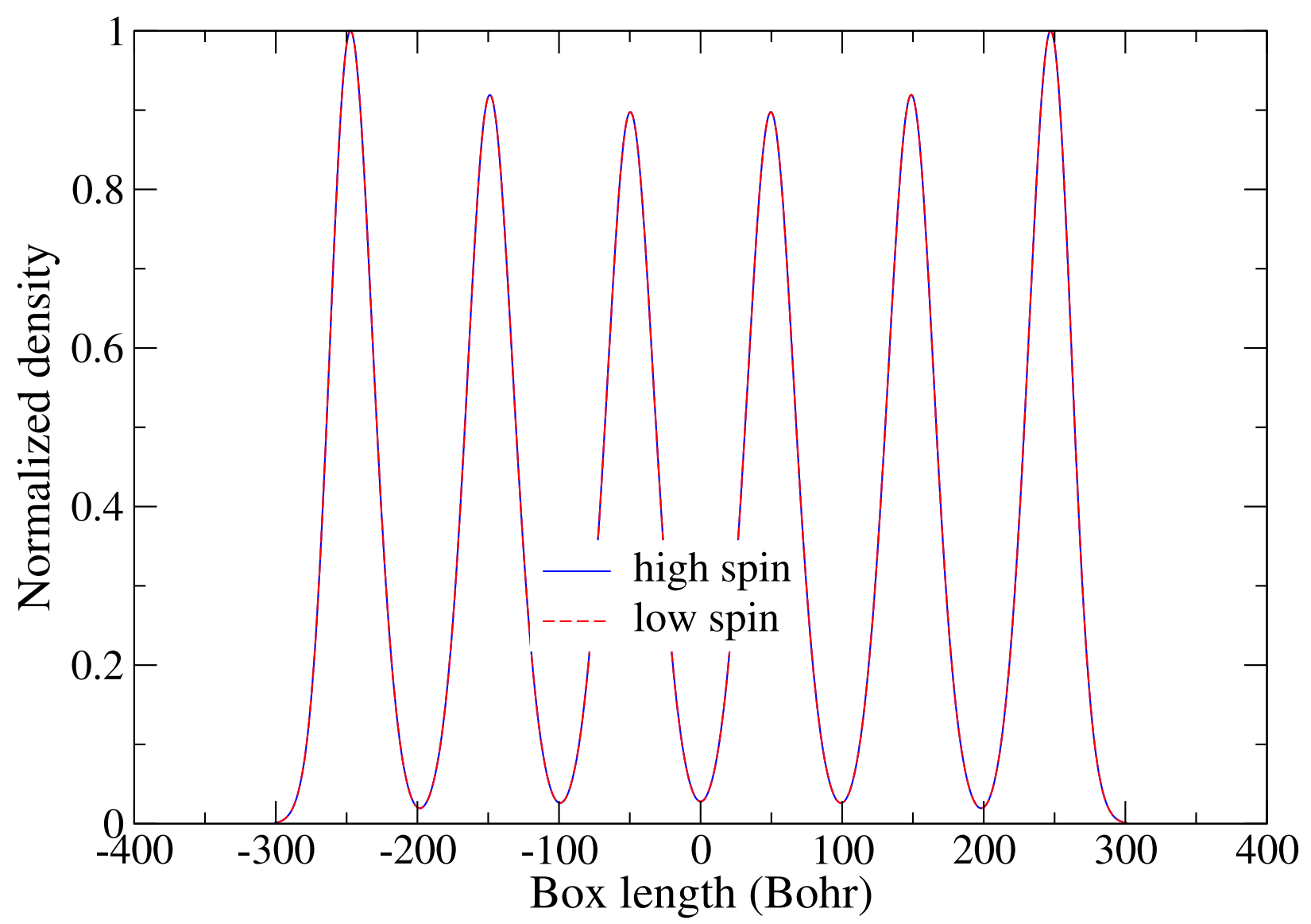

Figure 6. The electron density for $d=100.0$ Bohr. 


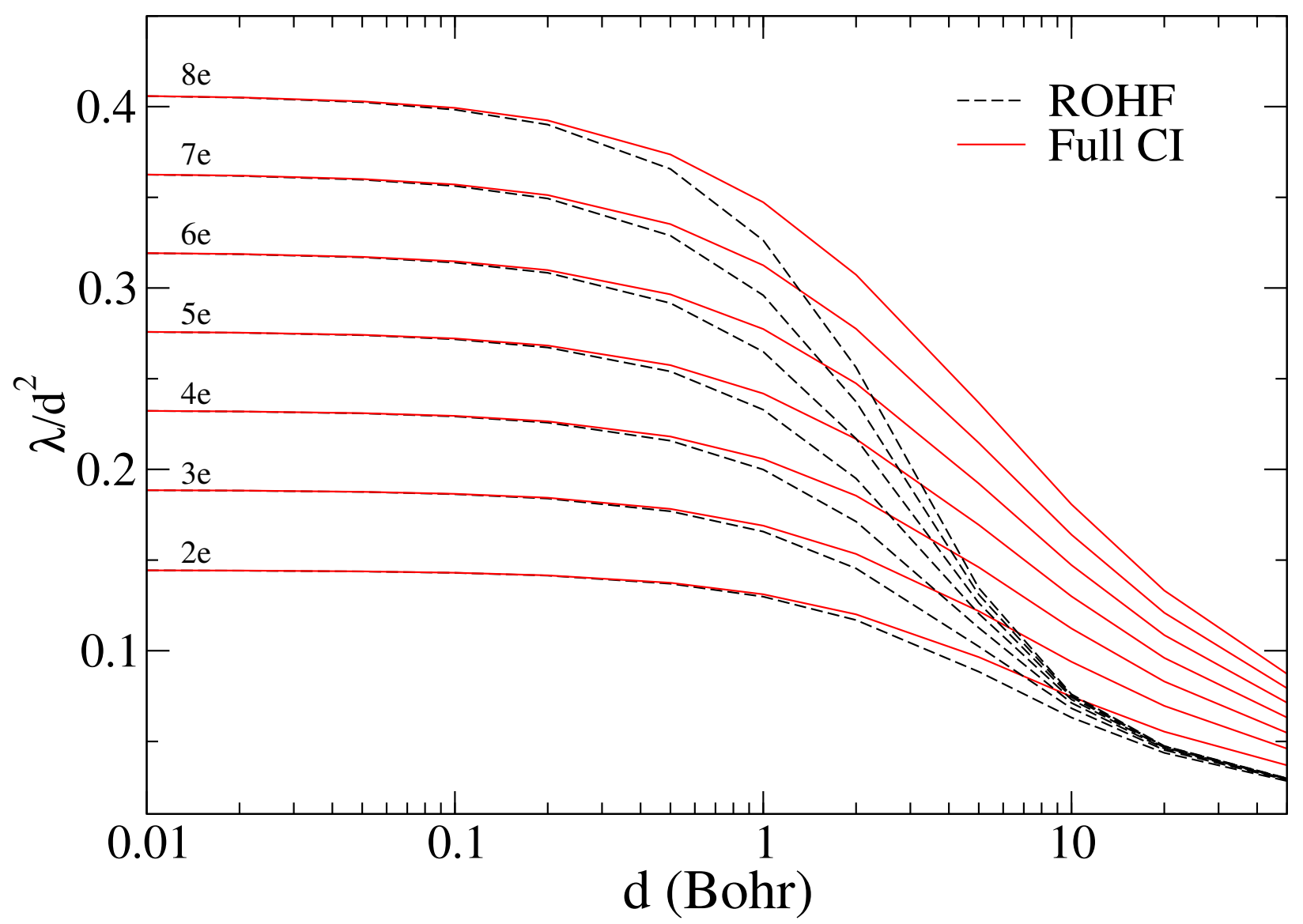

Figure 7. The scaled HS localization tensor $\lambda / d^{2}$ as a function of the mean distance $d$ (please note the logarithmic scale). 


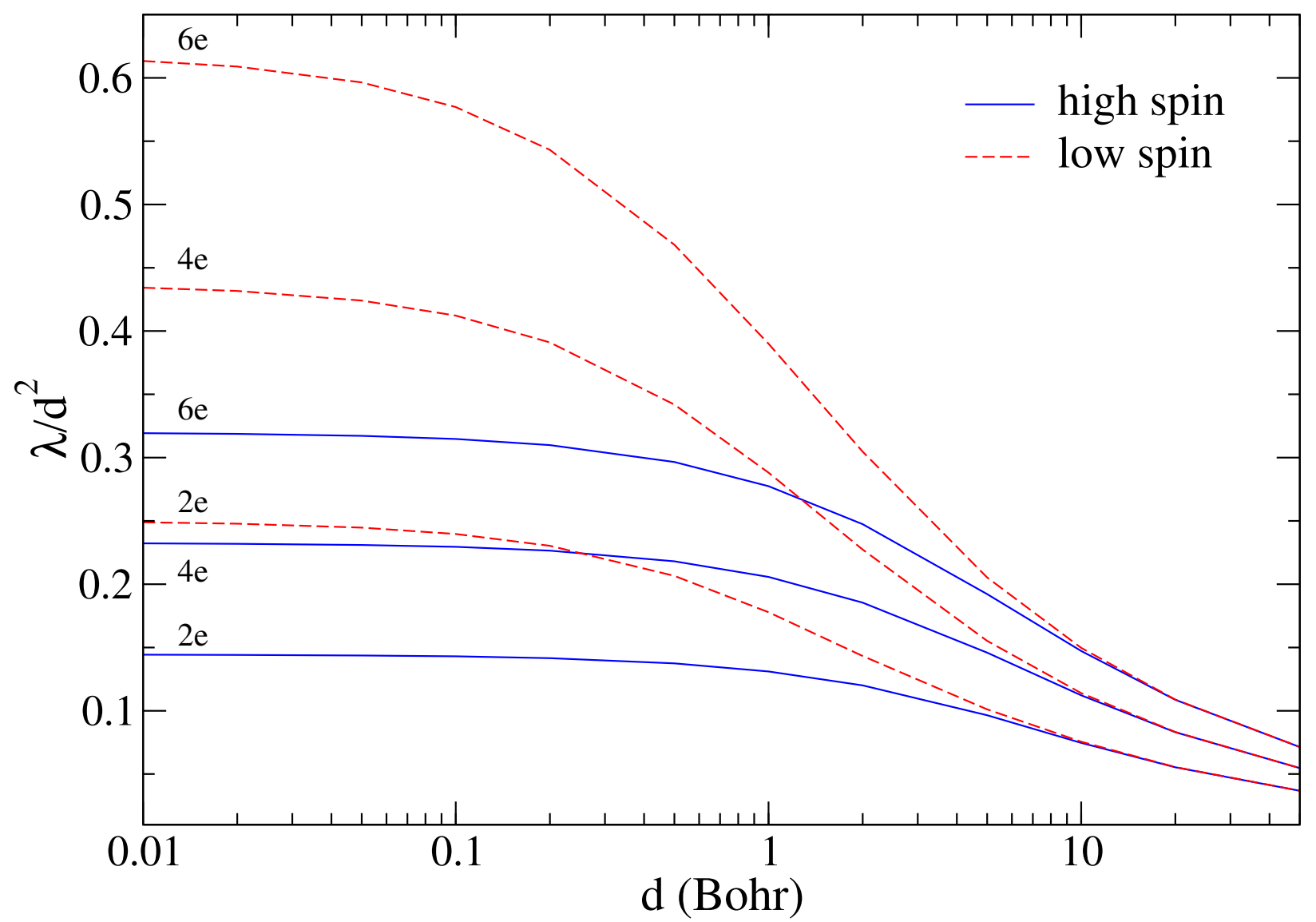

Figure 8. The scaled FCI localization tensor $\lambda / d^{2}$ as a function of the mean distance $d$ (please note the logarithmic scale). 


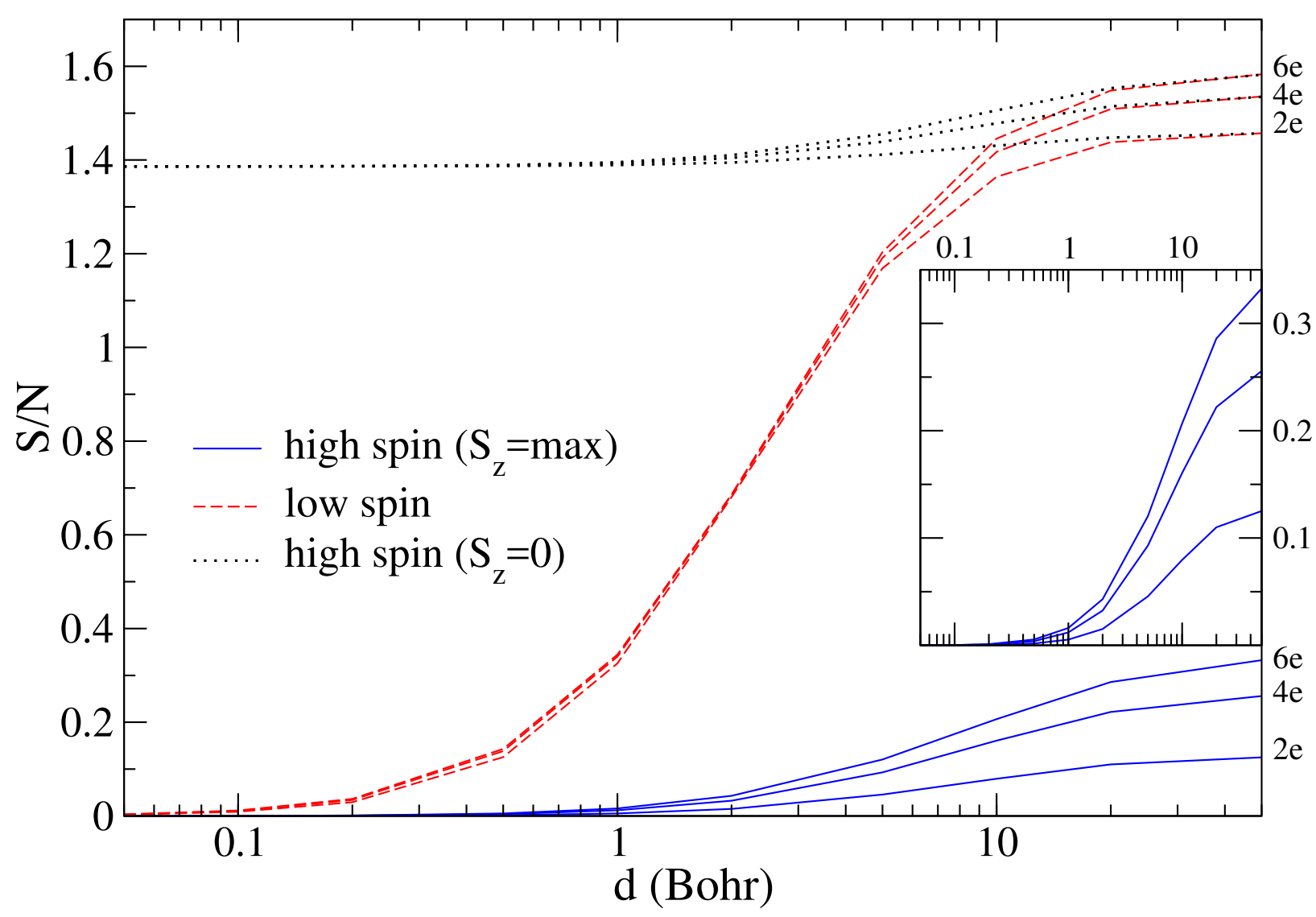

Figure 9. The total electronic entropy per electron $S / N$ as a function of the mean distance $d$ (please note the logarithmic scale). Inset: a zoom of the entropy of the HS state $\left(S_{z}=\max \right)$. 


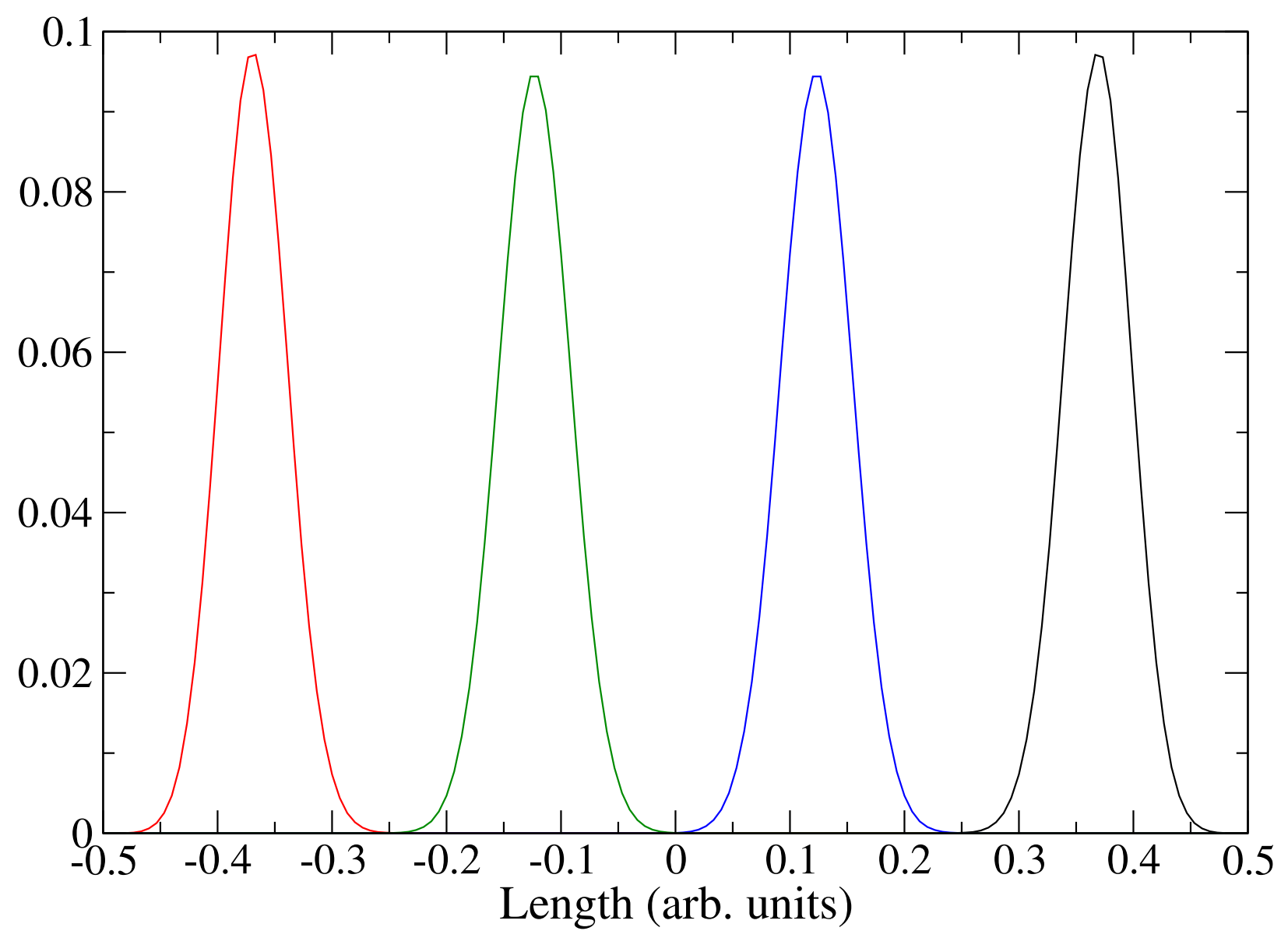

Figure 10. Four localized orbitals having the largest occupation numbers for the case $N=4$. Pairs of corresponding orbitals having $g$ and $u$ symmetries have been combined in order to obtain completely localized combinations. 


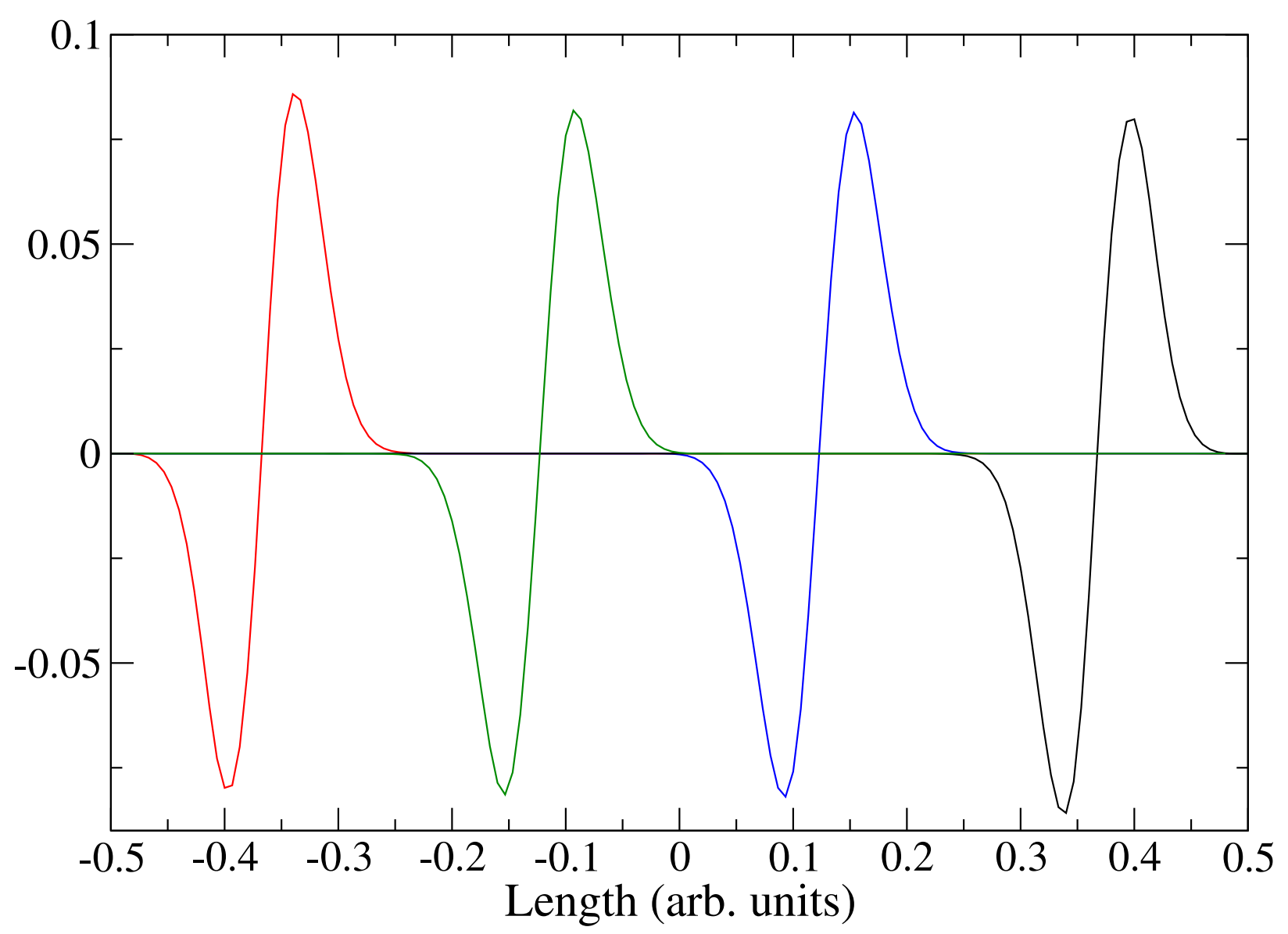

Figure 11. Four localized orbitals having the $5^{\text {th }}$ to $8^{\text {th }}$ largest occupation numbers for the case $N$ $=4$. Pairs of corresponding orbitals having $g$ and $u$ symmetries have been combined in order to obtain completely localized combinations. 


\section{REFERENCES}

${ }^{1}$ B. J. van Wees, H. van Houten, C. W. J. Beenakker, J. G. Williamson, L. P. Kouwenhoven, D. van der Marel, and C. T. Foxon, Phys. Rev. Lett. 60, 848 (1988).

${ }^{2}$ D. A. Wharam, T. J. Thornton, R. Newbury, M. Pepper, H. Ahmed, J. E. F. Frost, D. G. Hasko, D. C. Peacock, D. A. Ritchie, and G. A. C. Jones, J. Phys. C 21, L209 (1988).

${ }^{3}$ K. J. Thomas, J. T. Nicholls, M. Y. Simmons, M. Pepper, D. R. Mace, and D. A. Ritchie, Phys. Rev. Lett. 77, 135 (1996).

${ }^{4}$ K. J. Thomas, J. T. Nicholls, N. J. Appleyard, M. Y. Simmons, M. Pepper, D. R. Mace, W. R. Tribe, and D. A. Ritchie, Phys. Rev. B 58, 4846 (1998).

${ }^{5}$ S.-i. Tomonaga, Progress of Theoretical Physics 5, 544 (1950).

${ }^{6}$ J. M. Luttinger, Journal of Mathematical Physics 4, 1154 (1963).

${ }^{7}$ F. D. M. Haldane, Journal of Physics C: Solid State Physics 14, 2585 (1981).

${ }^{8}$ O. Auslaender, H. Steinberg, A. Yacoby, Y. Tserkovnyak, B. Halperin, K. Baldwin, L. Pfeiffer, and K. West, Science 308, 88 (2005).

${ }^{9}$ H. Steinberg, O. M. Auslaender, A. Yacoby, J. Qian, G. A. Fiete, Y. Tserkovnyak, B. I. Halperin, K. W. Baldwin, L. N. Pfeiffer, and K. W. West, Phys. Rev. B 73, 113307 (2006).

${ }^{10}$ V. V. Deshpande and M. Bockrath, Nat. Phys. 4, 314 (2008).

${ }^{11}$ J. M. Taylor and T. Calarco, Phys. Rev. A 78, 062331 (2008).

${ }^{12}$ E. Wigner, Phys. Rev. 46, 1002 (1934).

${ }^{13}$ J. Jang, B. M. Hunt, L. N. Pfeiffer, K. W. West, and R. C. Ashoori, Nature Physics 13, $340(2016)$.

${ }^{14}$ S. Pecker, F. Kuemmeth, A. Secchi, M. Rontani, D. Ralph, P. L. McEuen, and S. Ilani, Nature Physics 9, 576 (2013).

${ }^{15}$ L. Calmels and A. Gold, Phys. Rev. B 56, 1762 (1997).

${ }^{16}$ S. Bednarek, B. Szafran, T. Chwiej, and J. Adamowski, Phys. Rev. B 68, 045328 (2003).

${ }^{17}$ P. F. Loos and P. M. W. Gill, Phys. Rev. Lett. 108, 083002 (2012).

${ }^{18}$ P. F. Loos and P. M. W. Gill, J. Chem. Phys. 138, 164124 (2013).

${ }^{19}$ G. F. Giuliani and G. Vignale, Quantum theory of the electron liquid (Cambridge University Press) Chap. Appendix A.

${ }^{20}$ M. Rontani, C. Cavazzoni, D. Bellucci, and G. Goldoni, The Journal of Chemical Physics 124, 124102 (2006). 
${ }^{21}$ F. Malet, A. Mirtschink, J. C. Cremon, S. M. Reimann, and P. Gori-Giorgi, Phys. Rev. B 87, 115146 (2013).

${ }^{22}$ R. Resta, Rev. Mod. Phys. 66, 899 (1994).

${ }^{23}$ R. Resta, J. Chem. Phys. 124, 104104 (2006).

${ }^{24}$ W. Kohn, Phys. Rev. 133, A171 (1964).

${ }^{25}$ E. Kudinov, Fizika Tvergodo Tela 33, 2306 (1991).

${ }^{26}$ V. Vetere, A. Monari, G. L. Bendazzoli, S. Evangelisti, and B. Paulus, J. Chem. Phys. 128, 024701 (2008).

${ }^{27}$ G. L. Bendazzoli, S. Evangelisti, and A. Monari, International Journal of Quantum Chemistry 111, 3416 (2011).

${ }^{28}$ E. Giner, G. L. Bendazzoli, S. Evangelisti, and A. Monari, J. Chem. Phys. 138, 074315 (2013).

${ }^{29}$ A. Monari, G. L. Bendazzoli, and S. Evangelisti, J. Chem. Phys. 129, 134104 (2008).

${ }^{30}$ S. Evangelisti, G. L. Bendazzoli, and A. Monari, Theoretical Chemistry Accounts 126, 257 (2010).

${ }^{31}$ G. L. Bendazzoli, S. Evangelisti, A. Monari, and R. Resta, J. Chem. Phys 133, 064703 (2010).

${ }^{32}$ G. L. Bendazzoli, S. Evangelisti, and A. Monari, Int. J. Quantum Chemi. 112, 653 (2012).

${ }^{33}$ M. El Khatib, O. Brea, E. Fertitta, G. L. Bendazzoli, S. Evangelisti, T. Leininger, and B. Paulus, Theoretical Chemistry Accounts 134, 29 (2015).

${ }^{34}$ E. Fertitta, M. E. Khatib, G. L. Bendazzoli, B. Paulus, S. Evangelisti, and T. Leininger, J. Chem. Phys. 143, 244308 (2015).

${ }^{35}$ S. D. Sabatino, J. A. Berger, L. Reining, and P. Romaniello, J. Chem. Phys. 143, 024108 (2015).

${ }^{36}$ P. Gori-Giorgi and P. Ziesche, Phys. Rev. B 66, 235116 (2002).

${ }^{37}$ A. D. Gottlieb and N. J. Mauser, Phys. Rev. Lett. 95, 123003 (2005).

${ }^{38}$ L. Brooke, A. Diaz-Marquez, S. Evangelisti, T. Leininger, and J. A. Berger, Unpublished.

${ }^{39}$ G. L. Bendazzoli and S. Evangelisti, J. Chem. Phys. 98, 3141 (1993).

${ }^{40}$ L. Gagliardi, G. L. Bendazzoli, and S. Evangelisti, J. Comput. Chem. 18, 1329 (1997).

${ }^{41}$ NEPTUNUS is a Quantum-Chemistry FORTRAN code for the calculation of FCI and CAS-CI energies and properties written by G.L. Bendazzoli and S. Evangelisti, with contributions from L. Gagliardi, E. Giner, A. Monari, F. Passerini and M. Verdicchio. 
${ }^{42}$ H.-J. Werner, P. J. Knowles, G. Knizia, F. R. Manby, and M. Schütz, Wiley Interdisciplinary Reviews: Computational Molecular Science 2, 242 (2012).

${ }^{43}$ MOLPRO, version 2015.1, a package of ab initio programs, H.-J. Werner, P. J. Knowles, G. Knizia, F. R. Manby, M. Schütz, P. Celani, W. Györffy, D. Kats, T. Korona, R. Lindh, A. Mitrushenkov, G. Rauhut, K. R. Shamasundar, T. B. Adler, R. D. Amos, A. Bernhardsson, A. Berning, D. L. Cooper, M. J. O. Deegan, A. J. Dobbyn, F. Eckert, E. Goll, C. Hampel, A. Hesselmann, G. Hetzer, T. Hrenar, G. Jansen, C. Köppl, Y. Liu, A. W. Lloyd, R. A. Mata, A. J. May, S. J. McNicholas, W. Meyer, M. E. Mura, A. Nicklass, D. P. O’Neill, P. Palmieri, D. Peng, K. Pflüger, R. Pitzer, M. Reiher, T. Shiozaki, H. Stoll, A. J. Stone, R. Tarroni, T. Thorsteinsson, and M. Wang, see http://www.molpro.net.

${ }^{44}$ K. Aidas, C. Angeli, K. L. Bak, V. Bakken, R. Bast, L. Boman, O. Christiansen, R. Cimiraglia, S. Coriani, P. Dahle, E. K. Dalskov, U. Ekström, T. Enevoldsen, J. J. Eriksen, P. Ettenhuber, B. Fernández, L. Ferrighi, H. Fliegl, L. Frediani, K. Hald, A. Halkier, C. Hättig, H. Heiberg, T. Helgaker, A. C. Hennum, H. Hettema, E. Hjertenæs, S. Høst, I.-M. Høyvik, M. F. Iozzi, B. Jansík, H. J. A. Jensen, D. Jonsson, P. Jørgensen, J. Kauczor, S. Kirpekar, T. Kjærgaard, W. Klopper, S. Knecht, R. Kobayashi, H. Koch, J. Kongsted, A. Krapp, K. Kristensen, A. Ligabue, O. B. Lutnæs, J. I. Melo, K. V. Mikkelsen, R. H. Myhre, C. Neiss, C. B. Nielsen, P. Norman, J. Olsen, J. M. H. Olsen, A. Osted, M. J. Packer, F. Pawlowski, T. B. Pedersen, P. F. Provasi, S. Reine, Z. Rinkevicius, T. A. Ruden, K. Ruud, V. V. Rybkin, P. Sałek, C. C. M. Samson, A. S. de Merás, T. Saue, S. P. A. Sauer, B. Schimmelpfennig, K. Sneskov, A. H. Steindal, K. O. Sylvester-Hvid, P. R. Taylor, A. M. Teale, E. I. Tellgren, D. P. Tew, A. J. Thorvaldsen, L. Thøgersen, O. Vahtras, M. A. Watson, D. J. D. Wilson, M. Ziolkowski, and H. Ågren, Wiley Interdisciplinary Reviews: Computational Molecular Science 4, 269 (2014).

${ }^{45}$ H. Werner and P. J. Knowles, J. Chem. Phys. 82, 5053 (1985).

${ }^{46}$ P. J. Knowles and H.-J. Werner, Chem. Phys. Lett. 115, 259 (1985).

${ }^{47}$ J. Cioslowski, Phys. Rev. A 43, 1223 (1991).

${ }^{48}$ E. Valderrama, E. V. Ludeña, and J. Hinze, J. Chem. Phys. 106, 9227 (1997).

${ }^{49}$ E. Valderrama, E. V. Ludeña, and J. Hinze, J. Chem. Phys. 110, 2343 (1999).

${ }^{50}$ E. Ramos-Cordoba, P. Salvador, and E. Matito, Phys. Chem. Chem. Phys. 18, 24015 (2016). 